\title{
Development of an in vitro model to test antifibrotic drugs on primary human liver myofibroblasts
}

\author{
Lynda Aoudjehane ${ }^{1,2,3}$, Pierre-Yves Boelle ${ }^{4}$, Grégoire Bisch ${ }^{1}$, Rolland Delelo ${ }^{2,3}$, François Paye ${ }^{2,5}$, Olivier Scatton ${ }^{2,3,6}$, \\ Chantal Housset ${ }^{2,3}$, Jérôme Becquart ${ }^{1}$, Yvon Calmus ${ }^{2,3,7}$ and Filomena Conti ${ }^{2,3,7}$
}

We have developed a culture model to assess antifibrotic drugs using normal human liver myofibroblasts (HLMFs) obtained from 31 subjects. Activation was evaluated in terms of $a$-smooth muscle actin ( $a$-SMA) and collagen 1 (Coll1) expression using RT-PCR, and proliferation as the uptake of 5-ethynil-2'-deoxyuridine. Under analysis of variance, between-subject differences accounted for $70 \%$ of all variability and inter-experiment differences for $30 \%$. The sensitivity of the model was determined by quantifying the effects in terms of relative expression, which were $0.74 \pm 0.03$ for cyclosporine A (CsA) and $2.4 \pm 0.10$ for transforming growth factor-beta (TGF- $\beta)(P<0.0001$ vs no treatment) for $a$-SMA expression. Inter-subject variations in $a$-SMA and Coll1 expression enabled the classification of subjects as potentially low or high fibrosers. Finally, we observed that pirfenidone (which has beneficial effects in vivo) significantly reduced the expressions of $a$-SMA and Coll1, whereas the angiotensin-converting enzyme inhibitor losartan (which has no effect in vivo) had no significant effect. Our model may thus detect the antifibrotic properties of drugs. Antifibrotic drugs with promising clinical relevance could possibly be selected using a bank of HLMFs from high fibrosers.

Laboratory Investigation (2016) 96, 672-679; doi:10.1038/labinvest.2016.36; published online 7 March 2016

Fibrosis is characterized by an abnormal accumulation of extracellular matrix (ECM) components, irrespective of the organ or disease considered. It is the consequence of inflammatory and necrotic processes and ultimately leads to organ function failure. ${ }^{1}$ In the liver, fibrosis progresses toward cirrhosis, a condition responsible for most liver transplants and deaths from liver diseases. ${ }^{2}$ Very few drugs with proven antifibrotic effects have yet been made available. One such drug is pirfenidone, which was recently shown to reduce lung fibrosis ${ }^{3,4}$ and improve the prognosis of patients with idiopathic forms of this condition. Some other drugs have been tested in patients with liver fibrosis, such as angiotensin-converting enzyme inhibitors, colchicine or PPAR- $\delta$ agonists, but without achieving any significant improvement. ${ }^{5-7}$ There are several reasons for this lack of treatments for fibrotic liver disorders: (a) liver fibrosis is usually a very slow process that develops over decades, thus making the design of clinical trials particularly difficult; (b) only a minority of patients with liver diseases, whatever their cause (eg, alcoholic, metabolic or viral), actually develop severe fibrosis and cirrhosis, suggesting the presence in a given human population of both high- and low-rate 'fibrosers' ${ }^{5,8-11}$ and (c) most of the drugs tested so far probably exerted modest antifibrotic effects that were difficult to document during short-term trials.

Preclinical models of liver fibrosis have mainly been developed in animals, and include bile duct ligation, chronic intoxication with hepatotoxic drugs or $\mathrm{mdr} 2$ deficiency. However, these models are not necessarily representative of the human pathophysiology. They are costly and raise ethical issues relative to animal experimentation. In vitro cell models represent an alternative and complementary approach. We previously developed a culture model of human liver myofibroblasts (HLMFs), ${ }^{12}$ which offers direct access to ECM-producing cells in the liver as the principal targets of antifibrotic drugs, and could enable the rapid testing of a large number of candidate drugs. Based on this HLMF model, we have now developed an in vitro test that could be used to predict the antifibrotic properties of new compounds for the use in human liver disease.

\footnotetext{
${ }^{1}$ Human HepCell, Faculté de Médecine Pierre et Marie Curie, Site Saint-Antoine, Paris, France; ${ }^{2}$ Sorbonne Universités, UPMC University Paris 06, Paris, France; ${ }^{3}$ INSERM, UMR_S 938, CDR Saint-Antoine, Paris, France; ${ }^{4}$ AP-HP, Hôpital Saint Antoine, Unité de Biostatistique, Paris, France; ${ }^{5}$ AP-HP, Hôpital Saint Antoine, Service de Chirurgie Digestive, Paris, France; ${ }^{6}$ AP-HP, Hôpital Pitié-Salpêtrière, Servie de Chirurgie Digestive et Transplantation Hépatique, Paris, France and ${ }^{7}$ AP-HP, Hôpital Pitié-Salpêtrière, Unité de Transplantation Hépatique, Paris, France

Correspondence: Dr L Aoudjehane, PhD, Human HepCell, CDR-Inserm UPMC UMRS-938, Faculté de Médecine Pierre et Marie Curie-Saint Antoine, 27 rue de Chaligny, Paris 75012, France.

E-mail: Iynda.aoudjehane@inserm.f

Received 16 September 2015; revised 28 December 2015; accepted 11 January 2016
} 
However, with such a complex assay, the sources of variance are numerous and need to be assessed in order to define the best set-up. Indeed, human liver myofibroblasts are obtained from patients undergoing partial hepatectomy, whose response to antifibrotic drugs may vary. Further, using these cells in separate cultures/batches adds further variance, which may be further exacerbated by the final PCR measurement. Apportioning variability to these sources will be critical to clarify how many replicates, and of what type, need to be obtained. As a by-product, it will be possible to identify 'high' and 'low' responders among patients, and, to qualify for future use, only those whose response is likely to change during the evaluation of a new compound. Below, we describe this assessment and provide a first example regarding the evaluation of a new compound.

\section{MATERIALS AND METHODS Liver Samples}

All liver tissue specimens were obtained during surgical procedures carried out in Paris Public Hospitals (AP-HP) in France. All samples were obtained with anonymised and no-opposition statements from the patients. This procedure was approved by an Ethics Committee (CPP Ile de France III) and by the French Ministry of Health (Ref COL 2929 and COL 2930).

\section{HLMF Preparations}

Liver tissue specimens were obtained from 31 adult patients undergoing partial hepatectomy to treat metastases of colorectal cancer. All patients were seronegative for HCV, HBV and HIV. They were aged $55 \pm 6$ years (mean \pm s.d.) and included 17 males and 14 females. Histological evaluations were considered to be normal. Cell isolation was accomplished in compliance with French ethical guidelines, using an established method. ${ }^{12}$ The liver fragments (10-30 g) were initially perfused for $20 \mathrm{~min}$ with a prewarmed $\left(37^{\circ} \mathrm{C}\right)$ calcium-free buffer supplemented with $5 \mathrm{mM}$ ethylene glycol tetraacetic acid (EGTA) (Sigma Aldrich; Saint-Quentin Fallavier, France), followed by perfusion with a prewarmed $\left(37^{\circ} \mathrm{C}\right)$ buffer containing $6 \mathrm{mM}$ calcium $(\mathrm{CaCl} 2)$ (Sigma Aldrich) and $0.05 \%$ collagenase $(5 \mathrm{mg} / \mathrm{ml})$ (Sigma Aldrich), for $15 \mathrm{~min}$. The liver fragments were then gently shaken in wash medium to free the liver cells, and the resulting suspension was filtered through a gauze-lined funnel before the cells were centrifuged at low speed. Primary human hepatocytes (PHH) were collected from the pellet and used in primary culture, as previously described. ${ }^{13}$ The HLMFs were then obtained by gradient centrifugation, as described elsewhere. ${ }^{12,13}$ After 1 week of primary culture through several passages, all cells had a myofibroblast-like appearance and stained positive for vimentin and $\alpha$-smooth muscle actin (Dako, Les Ulis, France). During all subsequent experiments, the HLMFs were used at passage 4 .

\section{Characterization of HLMFs}

\section{Flow Cytometry}

The expressions of CD90 (fibroblasts), CD31 (endothelial cells), CD14 (macrophages) and $\alpha$-SMA (myofibroblasts) were measured by flow cytometry analysis as previously described. ${ }^{13}$ HLMFs were released from culture plates by trypsination, centrifuged, re-suspended in PBS $\left(3 \times 10^{5}\right.$ cells/ $\mathrm{ml}$ ) and then washed. The HLMFs were then fixed with $4 \%$ paraformaldehyde (PFA). A multicolor flow cytometry using antibodies with directly-conjugated fluorochromes was used, and the cells were stained with a cocktail of conjugated anti-membrane antibodies: anti-human CD31-PE (dilution 1/20; Beckman Coulter, France), anti-human CD14-APC alexa fluor 750 (dilution 1/20; Beckman Coulter), anti-human CD90-PC5 (dilution 1/20; Beckman Coulter) or with a cocktail of isotypic antibodies specific to each antibody used (dilution 1/20; Beckman Coulter) for $45 \mathrm{~min}$ at $4{ }^{\circ} \mathrm{C}$. The cells were then permeabilized with BD Cytofix/Cytoperm solution (BD Biosciences, Le Pont De Claix, France) for $20 \mathrm{~min}$ and stained with a non-conjugated anti-human $\alpha$-SMA (dilution $1 / 50$; Dako), for $45 \mathrm{~min}$ at $4{ }^{\circ} \mathrm{C}$. The cells were then incubated with FITC-labeled goat anti-mouse IgG (dilution 1/100; Caltag, Burlingame, CA, USA) or a FITC-conjugated isotype (mouse IgG) as a negative control for $30 \mathrm{~min}$ at $4{ }^{\circ} \mathrm{C}$. Cell staining was then determined using a BD LSR II flow cytometer (LSR II, UMS30-LUMIC Cytométrie Saint-Antoine) and analyzed using FACSDiva software (Version 6.1.1, BD Biosciences).

\section{LX2 Cell Line}

The LX2 cell line, an immortalized human hepatic stellate cell line, was obtained from Millipore S.A.S (Alsace, France) and cultured at $37^{\circ} \mathrm{C}$ in a humidified atmosphere containing $5 \% \mathrm{CO}_{2}$ in DMEM, which in turn contained $2 \%$ fetal calf serum (FCS), $100 \mathrm{U} / \mathrm{ml}$ penicillin and $100 \mathrm{mg} / \mathrm{ml}$ streptomycin.

\section{Cell Treatment}

HLMFs or the LX2 cell line were seeded on plastic in DMEM (10 or 2\% FCS, respectively) in 6-well plates or 96-well plates for the cell proliferation assay, at a density of $1.5 \times 10^{5}$ or $5 \times 10^{3}$ cells/well, respectively, and allowed to attach for $24 \mathrm{~h}$. Then, for all experiments, the medium was replaced with FCS-DMEM (1\% FCS) and the cells were cultured for $48 \mathrm{~h}$ without treatment (controls) or with recombinant human transforming growth factor-beta (TGF- $\beta)(10 \mathrm{ng} / \mathrm{ml}$; R\&D Systems, Lille, France), recombinant human platelet-derived growth factor (PDGF) $(10 \mathrm{ng} / \mathrm{ml}$; R\&D Systems), cyclosporine (CsA) $(1 \mu \mathrm{g} / \mathrm{ml}$, Novartis Pharma AG, Basel, Switzerland), pirfenidone (PFD) $(0.1,0.5,1$ or $2 \mathrm{mM}$; Sigma Aldrich) or losartan (LOS) $(1,10,50$ or $100 \mu \mathrm{M}$; Sigma). The cells were then detached from the culture plates by trypsination for further analyses. 


\section{Cytotoxicity Assay}

Cytotoxicity was determined by measuring lactate dehydogenase (LDH) release. After the treatment of cells with different concentrations of pirfenidone or losartan, the culture medium was collected and assayed using the LDH Cytotoxicity Detection Kit (Promega, Charbonnieres, France), according to the manufacturer's instructions. Background and negative controls were obtained by measuring LDH in the assay medium and unexposed cell medium, respectively. Total cellular LDH release was then measured in cell lysates obtained by treatment with $1 \%$ triton X-100 solution, and was used as the positive control. The data were calculated and expressed as a percentage cytotoxicity representing the mean of three independent experiments.

\section{Cell Proliferation Assay}

Cells were plated on 96-well plates at a density of $5 \times 10^{3}$ cells/ well. Twenty-four hours after plating, the cells were treated with PFD or LOS at different doses. Cell proliferation was determined by the uptake of 5-ethynil-2'-deoxyuridine (EdU) into DNA, using a Click-iT EdU microplate assay kit (Life technologies, Saint Aubin, France) according to the manufacturer's instructions. The cells were pulsed with EdU $(10 \mu \mathrm{M})$ for $18 \mathrm{~h}$, before the treatments were applied for $48 \mathrm{~h}$. The results were expressed as a percentage of maximum fluorescence (excitation/emission 568/585 nm) based on three replicates in preparations from four different patients.

\section{Quantitative RT-PCR Analyses of Collagen-1 and $a$-SMA}

Total RNA was prepared using the RNeasy minikit (Qiagen SA, Courtaboeuf, France) according to the manufacturer's recommendations. cDNA synthesis was performed for $90 \mathrm{~min}$ at $42^{\circ} \mathrm{C}$ in a reaction mixture containing two units of RNase inhibitor (Promega), three units of avian myeloblastosis virus reverse transcriptase (Promega), $120 \mathrm{ng}$ of random hexamer primers (Life Technologies) and $1 \mathrm{mM}$ DNTP (Promega) for 500 ng of total RNA.

Collagen-1 (Coll1) and $\alpha$-smooth muscle actin ( $\alpha$-SMA) gene expressions in HLMFs was analyzed by RT-PCR using the LightCycler 480 SYBR Green I Master $(2 \times$ con $)$ Kit (Roche, Grenoble, France), with LC480 instruments and technology (Roche Diagnostics). PCR amplification was performed in a total volume of $10 \mu \mathrm{l}$, containing $5 \mu \mathrm{l}$ Sybr green I Master Mix $(2 \times), 10 \mathrm{ng}$ of each primer (Sigma-Genosys Ltd; Supplementary Table S1) and $2 \mu \mathrm{l}$ cDNA (1/10). The PCR amplification protocol comprises one step of initial denaturation for $10 \mathrm{~min}$ at $94^{\circ} \mathrm{C}$, followed by 40 cycles involving denaturation $\left(95^{\circ} \mathrm{C}\right.$ for $\left.10 \mathrm{~s}\right)$, annealing for $10 \mathrm{~s}$ at $60^{\circ} \mathrm{C}$ and extension $\left(72^{\circ} \mathrm{C}\right.$ for $\left.10 \mathrm{~s}\right)$. The mRNA level was calculated by normalizing the threshold cycle (CT) of Coll1 or $\alpha$-SMA to the CT of the $28 \mathrm{~S}$ ribosomal RNA housekeeping gene, as described elsewhere, ${ }^{14}$ The primers used during this study were as previously described ${ }^{13}$ and purchased from Sigma-Genosys Ltd.

\section{Statistical Analysis}

We estimated the variance components of $\alpha$-SMA and Coll1 using a random model analysis of variance applied to the control experiments only. Variance was split into three nested components: between patients, between experiments (nested in patients) and between PCRs (nested within experiments). The relative importance of each component was determined by apportioning total variance as a function of its components. In this analysis, we pooled the results obtained with $\alpha$-SMA and collagen, because a preliminary analysis had shown that the variance breakdown was similar.

The effects of the drugs were estimated using a mixed model for analysis of variance with fixed effects for drugs and random effects for subjects, experiments and PCR, ie, assuming that the subject-specific drug effect could fluctuate around an overall response. Fixed effects were compared to zero using the log-likelihood ratio test. The variability between subjects was described by computing patientspecific drug effect responses over different experiments in the same patient. All analyses were performed using R software (v3.0).

\section{RESULTS}

\section{Characterization of HLMF}

The characterization of HLMF was performed using fourcolor flow cytometric analysis. The cell population was gated according to its isotype control (Figure 1). During these analyses, $92 \pm 4,1.3 \pm 0.5,0.7 \pm 0.3$ and $85 \pm 5 \%$ of the HLMF expressed CD90, CD31, CD14 and $\alpha$-SMA, respectively. Therefore, the majority of HLMF were CD90+CD31$\mathrm{CD} 14-$ and $\alpha$-SMA+. Figure 1 shows one representative experiment (out of 31) performed during this study.

\section{Analysis of Subjects, Experiments and PCR Variations}

Fibrogenesis was evaluated at the cell level in HLMF preparations from 31 subjects, in terms of both myofibroblastic differentiation as reflected by $\alpha$-SMA expression, and of ECM production as reflected by Coll1 production. Analysis of variance revealed that these parameters varied considerably between subjects. Between-subject differences accounted for $\sim 70 \%$ of all variability, inter-experiment differences for $30 \%$ and PCR repeats for $<1 \%$ regarding both $\alpha$-SMA and Coll1 expression levels (Figure 2).

To assess the sensitivity of our test in assessing the pro- or antifibrotic effects of drugs, we submitted the cell cultures to pro- and antifibrotic control treatments with TGF- $\beta$ and cyclosporine A (CsA), respectively. ${ }^{15,16}$ These treatments induced significant changes in myofibroblast differentiation and ECM production (Figure 3). Quantification of the effects of these drugs, expressed as fold changes, showed $0.38 \pm 0.02$ for CsA and $2.9 \pm 0.15$ for TGF- $\beta(P<0.0001$ vs control experiments without drugs in both cases) with respect to $\alpha$-SMA expression (Figure 3), and $0.74 \pm 0.03$ for CsA and $2.4 \pm 0.10$ for TGF- $\beta(P<0.0001$ vs control experiments without drugs in both cases) for Coll1 (Figure 3). 

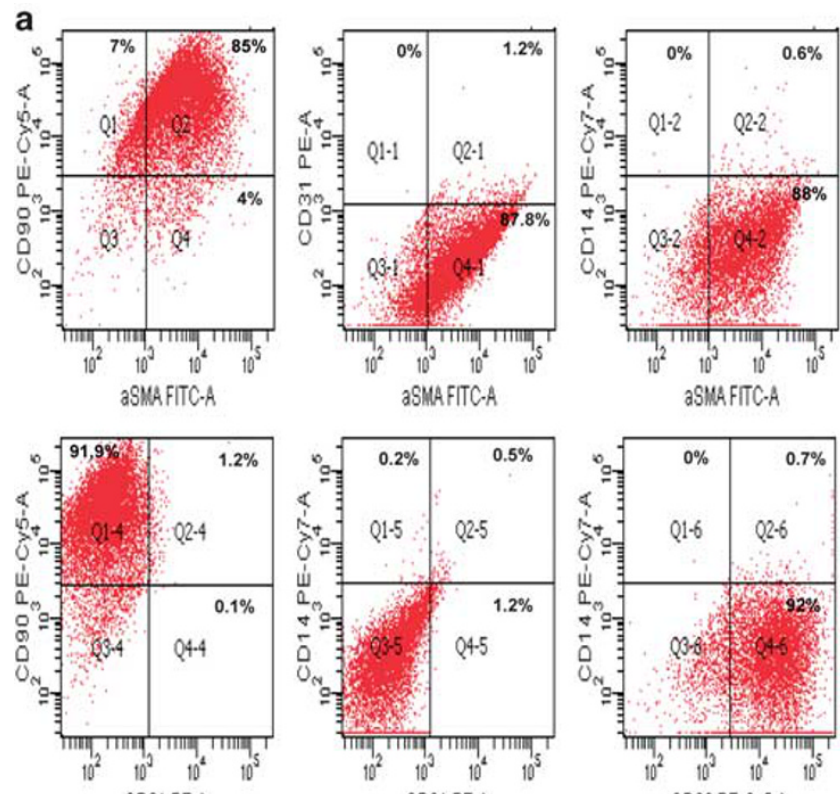

CD31 PE-A

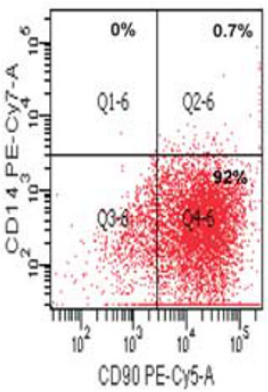

b
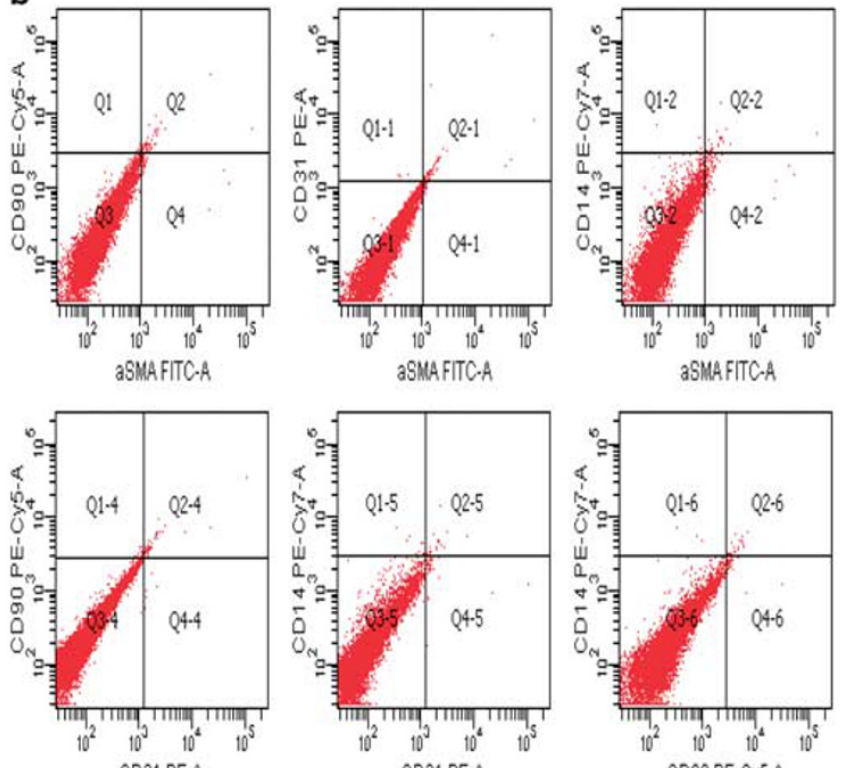

CD31 PE-A

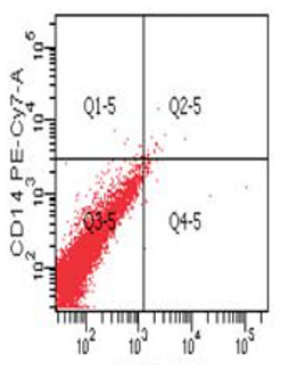

CD31 PE-A

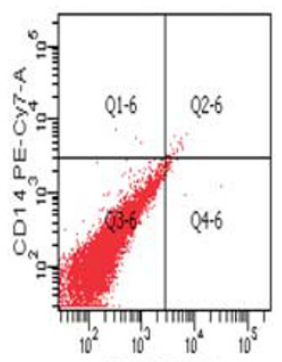

CD9O PE-C.5.A

Specific antibodies

Isotypic antibodies

Figure 1 Characterization of HLMF: detection of CD90, CD14, CD31 and a-SMA in HLMFs using multi-labeling by flow cytometry. HLMF were stained with a cocktail of mouse monoclonal antibodies (a) or negative controls (b) and then analyzed by flow cytometry. This figure shows 1 characteristic experiment out of the 31 performed.

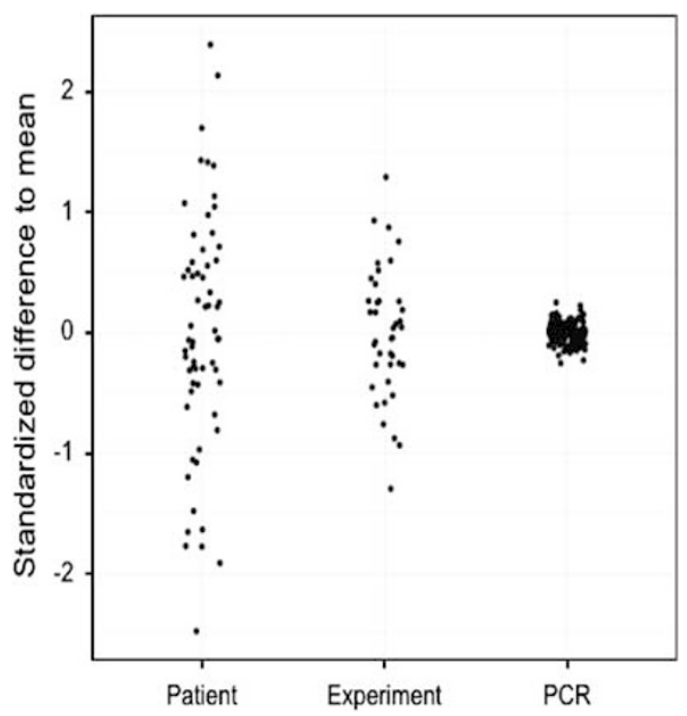

Figure 2 Components of variability in $a$-SMA and Coll1 transcription levels. Overall data were first normalized to mean 0 and variance 1. Differences from the mean, taking nesting into account (ie, within patients for experiments, within experiments for PCR) were then computed. Between-patient variability accounted for $\sim 70 \%$ of all variability, between-experiment variability for $30 \%$ and PCR repeats for $<1 \%$.

These latter analyses also provided information on the standard deviation of the treatment effect, which could be used to calculate the number of experiments and PCR repeats required to detect a given antifibrotic effect with predefined power. For both $\alpha$-SMA and Coll1, the standard deviation of the treatment estimate was $\sim 0.6$ cycles due to the experiment and 0.1 to the PCR. The number of experiments necessary to evidence a fold change of 0.7 ( $\Delta \Delta \mathrm{CT}$ effect of size 0.5$)$ with $80 \%$ power was therefore 13 , five for a fold change of 0.5 (size $1 \Delta \Delta \mathrm{CT}$ ) and four for a fold change of 0.3 (size 1.5 $\Delta \Delta \mathrm{CT}$ ). Repeating PCRs within experiments only marginally affected this result, beacuse most of the variance was between experiments.

It was also possible to determine the inter-subject variability of fibrogenesis (which may reflect the polymorphism of liver fibrogenesis in the human population) from our data (Figure 4). Individual changes in $\alpha$-SMA expression induced by TGF- $\beta$ or inhibited by CsA were determined for each individual. The between-subject coefficient of variation (s.d. to mean ratio) was $70-80 \%$ for both TGF- $\beta$ and CsA. Intra-subject reproducibility, measured by the intraclass correlation, was between 66 and $82 \%$. Using the results above, we computed that patients observed once within the lower tertile ('low-rate fibrosers', ie, the 33\% least responding) for $\alpha$-SMA expression after TGF- $\beta$ stimulation had a chance of between 60 and $80 \%$ of being confirmed as a low-rate fibroser in further replications, and a chance of $<10 \%$ of belonging to the highest tertile ('high-rate responders'). Three repeated observations in the lower range would make it more certain that a patient is a 'low-rate fibroser' (probability: 75-90\%). It should be stressed that there was no strong correlation between the responses to CsA and TGF- $\beta$ (Spearman correlation coefficient $r=0.2, P=0.17$ ). 


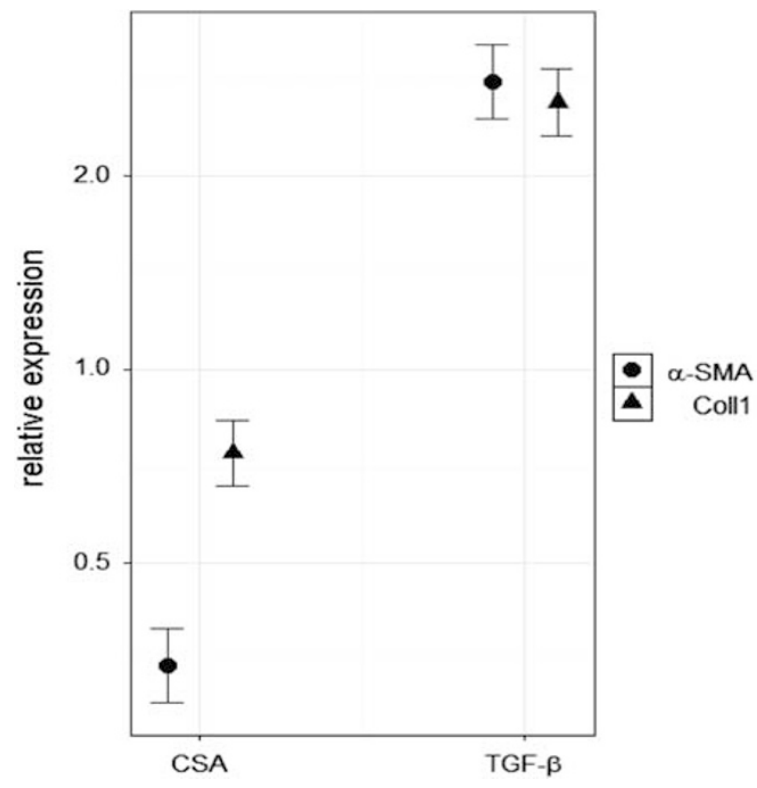

Figure 3 Effects of treatment with CSA or TGF- $\beta$ on $\alpha$-SMA and coll1 in 31 patients. Data are expressed in terms of $2^{-\Delta \Delta C T}$ and presented as mean \pm s.d.

\section{Antifibrotic Effects of Pirfenidone and Losartan on HLMF}

Finally, we used this model to test the potential antifibrotic effect of pirfenidone (PFD) (a drug used to treat idiopathic lung fibrosis) on liver fibrosis, and of losartan (LOS), the beneficial effects of which have previously been discussed in liver fibrosis. Using the $\alpha$-SMA and Coll1 expression data obtained in four patients (with one experiment per patient) to achieve the reliable detection of an antifibrotic effect of at least 0.5 cycle of $\Delta \Delta \mathrm{CT}$, we observed a significant effect with PFD concentrations $\geq 500 \mu \mathrm{M}(P<0.05)$ (Figure 5), while the expression of $\alpha$-SMA and Coll1 in HLMF treated with $2 \mathrm{mM}$ PFD was significantly decreased $(0.10 \pm 0.01 ; P<0.0001$ and $0.31 \pm 0.13 ; P=0.002$ respectively) (Figures $5 \mathrm{a}$ and $\mathrm{c}$ ). By contrast, LOS at doses up to $100 \mu \mathrm{M}$ had no significant effects on the expression of $\alpha$-SMA or Coll1 (Figures $5 \mathrm{~b}$ and d).

The inhibitory effect of PFD and LOS on HLMF proliferation was assessed from EdU incorporation. The proliferation of HLMFs was significantly stimulated with PDGF, but with PFD treatment it was markedly reduced as from a dose of $1 \mathrm{mM}$ (Figure 5e). By contrast, losartan did not display any suppressive effect on HLMF proliferation (Figure 5f). The PFD and LOS concentrations used during these experiments were similar to those previously used in in vitro models that had enabled detection of their pharmacological effects. ${ }^{17,18}$ Furthermore, no cytotoxicity was observed with the concentrations used during this study (Figures $5 \mathrm{~g}$ and $\mathrm{h}$ ).

We were then able to compare the inhibitory effect of PFD on HLMFs and the LX2 cell line, and showed that the half-maximal inhibitory concentrations $\left(\mathrm{IC}_{50}\right)$ of PFD on regarding the expression of $\alpha$-SMA and Coll1 and proliferation

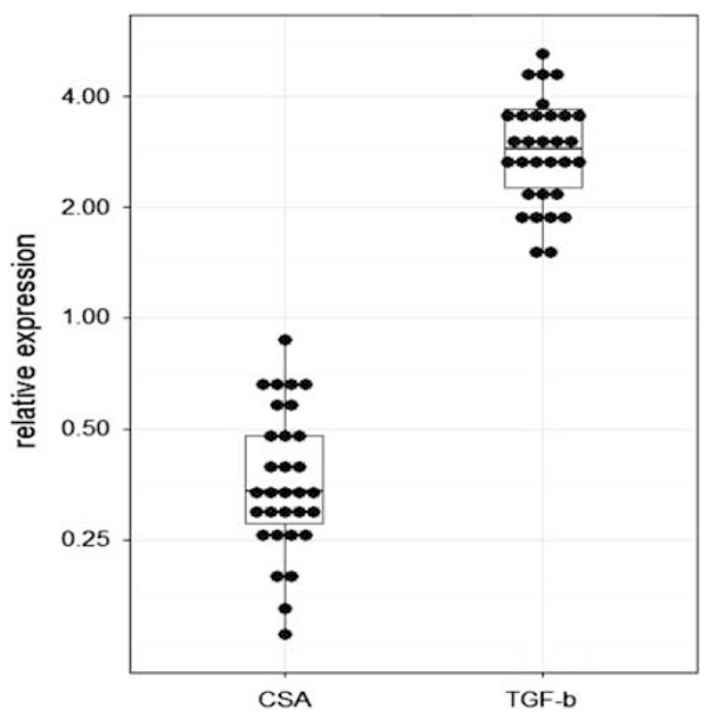

Figure 4 Between-patient variability of fibrogenesis. HLMF from 31 patients were treated with TGF- $\beta$ or CSA and analyzed for $\alpha$-SMA expression. Box corresponds to the kernel-based density estimator.

on HLMFs were 0.8 to $1.95 \mathrm{mM}, 6$ to $7 \mathrm{mM}$, and 1.42 to $3.4 \mathrm{mM}$, respectively. The $\mathrm{IC}_{50}$ values for PFD regarding the expression and proliferation of $\alpha$-SMA and Coll1 for the LX2 cell line were $0.68,1.00$ and $2.0 \mathrm{mM}$, respectively.

\section{DISCUSSION}

The main originality of our model is that it uses normal HLMFs which can offer direct access to ECM-producing cells in the liver as the principal targets of antifibrotic drugs, and could enable the rapid testing of a large number of candidate drugs. $\alpha$-SMA expression was chosen as a reliable marker for the activation of these cells. In vivo, $\alpha$-SMA overexpression in liver biopsies has proved to be associated with the rapid progression of fibrosis in humans. ${ }^{19,20}$ Coll1 was previously chosen as a marker of ECM production. The quantification of Coll expression in liver biopsies has also been employed extensively as a prognostic marker of cirrhosis. ${ }^{21,22}$ Matrix metalloproteinase-1 and 2, tissue inhibitor of matrix metalloproteinase (TIMP)-1 and fibronectin, have also been evaluated, but proved to be more inconsistently sensitive to the effects of CsA and TGF- $\beta$, possibly because all these molecules are produced by numerous cell types as well as HLMFs.

Liver fibrosis is clearly a heterogeneous process in humans: among individuals exposed to a known fibrosing processsuch as, chronic alcoholism, dysmetabolic syndrome or chronic hepatitis C-only $20-30 \%$ will go on to develop cirrhosis during the next 5-20 years, and the rate of fibrosis progression is markedly variable. It is thus possible to define rapid and low fibrosers. ${ }^{23,24}$ The polymorphism involved in this inter-patient variability may be expressed at several levels: the production and degradation of inflammatory mediators, a variability of HLMF activation or the production and 
a

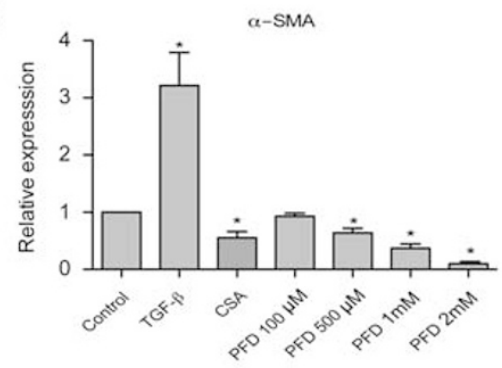

C

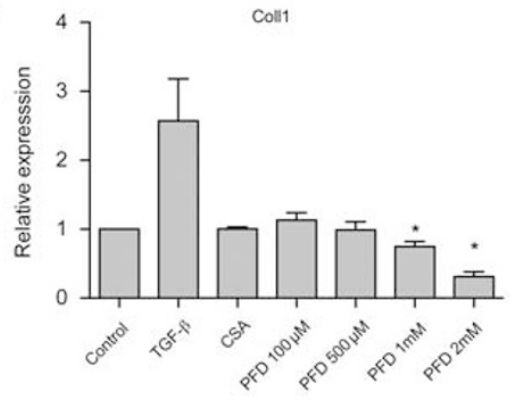

e

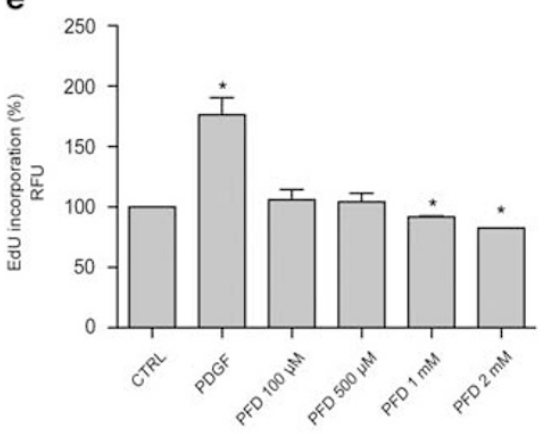

g

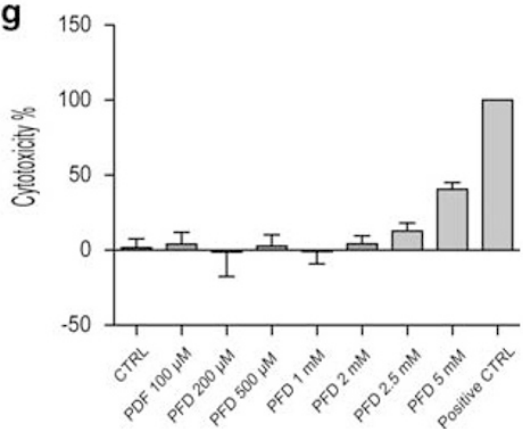

b

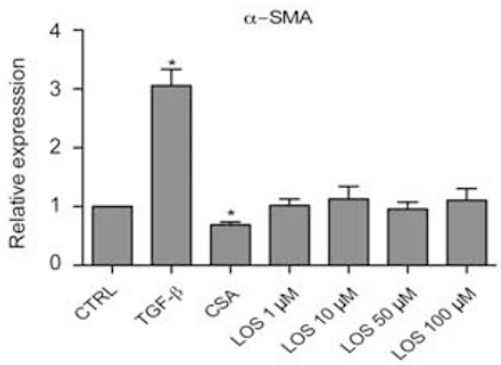

d

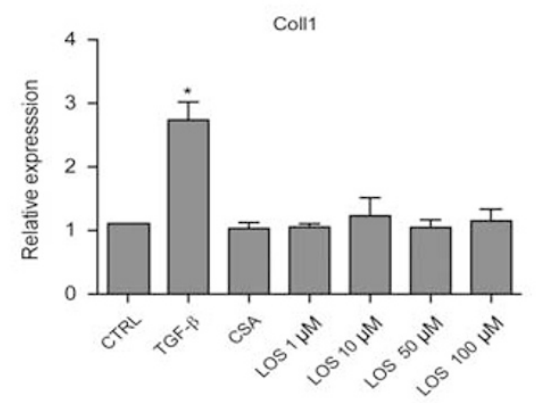

f

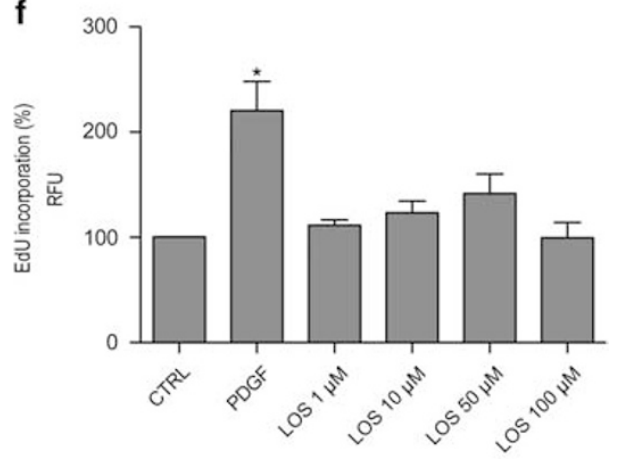

h

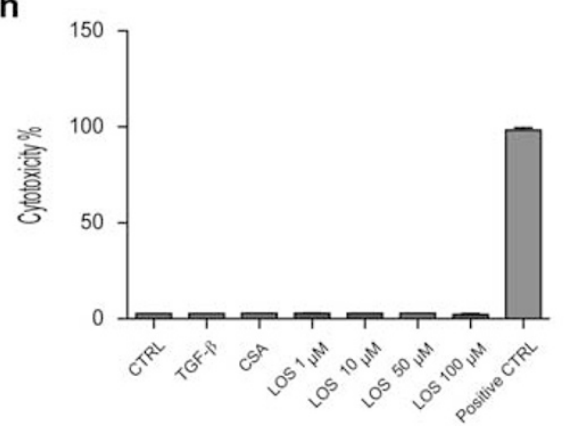

Figure 5 Effect of losartan (Los) and pirfenidone (PFD) on a-SMA ( $\mathbf{a}$ and $\mathbf{b}$ ) and Coll1 (c and $\mathbf{d}$ ) expression. Results are presented as the relative expression of Coll1 or $a$-SMA (by comparison with the control) using the value of $2^{-\Delta \Delta C T}$ and the results are mean \pm s.d. of four cell preparations, ${ }^{*} P \leq 0.05$ vs control. Effect of Los and PFD on HLMF proliferation. Results are presented as the percentage fluorescence after the uptake of 5-ethynil-2'deoxyuridine (EdU) (e and $\mathbf{f}$ ). Assessment of cytotoxicity of LOS and PFD on HLMF cells. The results are presented as the percentage of LDH release ( $\mathbf{g}$ and $\mathbf{h})$. All data are mean \pm s.d. of four cell preparations $\left({ }^{*} P \leq 0.05\right.$ vs control). Effect of Los and PFD on HLMF proliferation. Results are presented as the percentage fluorescence after the uptake of 5-ethynil-2'-deoxyuridine (EdU) (a and b). Assessment of cytotoxicity of LOS and PFD on HLMF cells. The results are presented as the percentage of LDH release ( $\mathbf{c}$ and $\mathbf{d})$. All data are mean \pm s.d. of four cell preparations $\left({ }^{*} P \leq 0.05\right.$ vs control).

degradation of ECM. ${ }^{11,25,26}$ Our results suggest that at least part of this inter-patient variability in fibrosis progression is dependent on the HLMF themselves; their activation and ECM production (in our case, evaluated from $\alpha$-SMA and
Coll1 transcription) and their proliferation vary between subjects, displaying a regular-Gaussian-distribution for the three parameters. We can therefore advance the hypothesis that subjects who spontaneously display higher activation 
and/or proliferation rates of their HLMF are predisposed to being rapid fibrosers, although our present experiments did not allow us to draw any conclusions, because the HLMF were obtained from patients undergoing surgery for metastases, without fibrosing disease and without significant liver fibrosis (data not shown).

The development of new drugs is often reliant on simple cell models that can test thousands of different compounds simultaneously. ${ }^{7}$ In the case of liver fibrosis, animal and human myofibroblast cell lines are available, but their functions differ from those of normal human cells. ${ }^{27,28} \mathrm{In}$ particular, the proliferation of cell lines is often unusually high, and their fibrotic parameters (activation and ECM production) remain unknown. When compared with myofibroblastic cell lines, our model was able to approach the human polymorphism of fibrogenesis, which is a mandatory requirement when trying to prove that a new compound could be efficient in high-rate fibrosers, who probably constitute the target for future antifibrotic treatment. We therefore decided to use normal HLMF from a bank, recently established using specimens from 31 subjects and gradually expanded since then, because these cells can easily be frozen. We used cells from unselected subjects for this study in order to address human polymorphism at the cell level. It has now become possible to select subjects from the high- and low-fibrogenesis tertiles in order to determine the potency of new antifibrotic drugs, which are mostly dedicated to treating patients with the highest risk of cirrhosis.

Our findings also suggest that detecting the antifibrotic properties of a new compound requires repeated experiments; only a standard duplicate or triplicate PCR, and the assessment of several subjects, will be relevant. Our model enables the detection of the antifibrotic effects of compounds with good reliability for effects as weak as 0.7 of $2^{-\Delta \Delta C T}$, which is therefore wholly sufficient regarding the effects of known activators or inhibitors of fibrogenesis, such as TGF- $\beta$ or $\mathrm{CsA}^{15,16,29,30}$ or of a drug with a proven antifibrotic effect such as PFD. ${ }^{3,18}$ Indeed, PFD was recently used for 2 years in 28 patients with chronic hepatitis $\mathrm{C}$ and was shown to improve inflammation, fibrosis and steatosis; ${ }^{31}$ furthermore, it was well tolerated at the dose used during the study $(1200 \mathrm{mg} /$ day), and none of the patients dropped out of the study because of the adverse effects of treatment. Angiotensin-converting enzyme inhibitors, which have been shown to exert antifibrotic properties in rodent models of liver fibrosis ${ }^{32}$ have no significant effects in humans. ${ }^{33-35}$ Interestingly, LOS had no effects in our hands, thus confirming the marked relevance of this model.

The $\mathrm{IC}_{50}$ of PFD on the three parameters used to assess the potential antifibrotic effect of this drug-the expression of $\alpha$-SMA and Coll1 and proliferation-were higher in HLMF than in the LX2 cell line. This suggests that testing new compounds on cell lines may overestimate their antifibrotic effect and lead to the development of drugs without clinical relevance. We suggest that cell lines could be used for initial screening before an evaluation on normal HLMF, obtained mainly from subjects with high spontaneous fibrosing parameters.

In the future, the availability of a gradually supplemented bank of HMLF may also help to determine biomarkers that are easily available in the blood to characterize high and low fibrosers (the latter being the only patients who require antifibrotic therapy), and for use in future trials on liver fibrosis. ${ }^{36}$ The availability of a gene signature has recently been proposed to develop new drugs for the treatment of lung fibrosis. $^{37}$

This HLMF-based model offers direct access to liver collagen-producing cells, which are a key factor in the pathogenesis of liver fibrosis. It may help to prove the mechanisms of action of future antifibrotic drugs, by analyzing the different components (proliferation, activation, ECM production) of fibrogenesis, and could be used to test drug candidates before their development enters its clinical phases. Future studies will be able to use a gradually supplemented bank of HLMFs from high fibrosers to help to select the antifibrotic drugs with the optimum clinical relevance.

Supplementary Information accompanies the paper on the Laboratory Investigation website (http://www.laboratoryinvestigation.org)

\section{DISCLOSURE/CONFLICT OF INTEREST}

The authors declare no conflict of interest.

1. Friedman SL, Sheppard D, Duffield JS, et al. Therapy for fibrotic diseases: nearing the starting line. Sci Transl Med 2013;5:167sr161.

2. Blachier $M$, Leleu $H$, Peck-Radosavljevic $M$, et al. The burden of liver disease in Europe: a review of available epidemiological data. J Hepatol 2013;58:593-608.

3. Peng $\mathrm{Y}$, Yang $\mathrm{H}$, Wang $\mathrm{N}$, et al. Fluorofenidone attenuates hepatic fibrosis by suppressing the proliferation and activation of hepatic stellate cells. Am J Physiol Gastrointest Liver Physiol 2014;306:G253-G263.

4. Conte E, Gili E, Fagone E, et al. Effect of pirfenidone on proliferation, TGF-beta-induced myofibroblast differentiation and fibrogenic activity of primary human lung fibroblasts. Eur J Pharm Sci 2014;58:13-19.

5. Rockey DC. Current and future anti-fibrotic therapies for chronic liver disease. Clin Liver Dis 2008;12:939-962, xi.

6. Inagaki Y, Higashiyama R, Higashi K. Novel anti-fibrotic modalities for liver fibrosis: molecular targeting and regenerative medicine in fibrosis therapy. J Gastroenterol Hepatol 2012;27:85-88.

7. Rosenbloom J, Mendoza FA, Jimenez SA. Strategies for anti-fibrotic therapies. Biochim Biophys Acta 2013;1832:1088-1103.

8. Anstee QM, Day CP. The genetics of NAFLD. Nat Rev Gastroenterol Hepatol 2013;10:645-655.

9. Roman S, Zepeda-Carrillo EA, Moreno-Luna LE, et al. Alcoholism and liver disease in Mexico: genetic and environmental factors. World J Gastroenterol 2013;19:7972-7982.

10. Zain SM, Mohamed R, Cooper DN, et al. Genome-wide analysis of copy number variation identifies candidate gene loci associated with the progression of non-alcoholic fatty liver disease. PLoS One 2014;9: e95604.

11. Machado MV, Janeiro A, Miltenberger-Miltenyi G, et al. Genetic polymorphisms of proangiogenic factors seem to favor hepatocellular carcinoma development in alcoholic cirrhosis. Eur J Gastroenterol Hepatol 2014;26:438-443.

12. Aoudjehane L, Pissaia A Jr, Scatton O, et al. Interleukin-4 induces the activation and collagen production of cultured human intrahepatic fibroblasts via the STAT-6 pathway. Lab Invest 2008;88:973-985. 
13. Aoudjehane L, Bisch G, Scatton O, et al. Infection of human liver myofibroblasts by hepatitis $C$ virus: a direct mechanism of liver fibrosis in hepatitis C. PLoS One 2015;10:e0134141.

14. Livak KJ, Schmittgen TD. Analysis of relative gene expression data using real-time quantitative PCR and the 2(-delta delta $C(T)$ ) method. Methods 2001;25:402-408.

15. Nakamuta M, Kohjima M, Fukushima M, et al. Cyclosporine suppresses cell growth and collagen production in hepatic stellate cells. Transplant Proc 2005;37:4598-4602.

16. Pissaia A Jr, Aoudjehane L, Ben Othman S, et al. Cyclosporine inhibits profibrotic effects of interleukin-4 and transforming growth factor beta on human intrahepatic fibroblasts cultured in vitro. Transplant Proc 2010;42:4343-4346.

17. Diop-Frimpong B, Chauhan VP, Krane S, et al. Losartan inhibits collagen I synthesis and improves the distribution and efficacy of nanotherapeutics in tumors. Proc Natl Acad Sci USA 2011;108:2909-2914.

18. Westra IM, Oosterhuis D, Groothuis GM, et al. The effect of antifibrotic drugs in rat precision-cut fibrotic liver slices. PLoS One 2014;9:e95462.

19. Gawrieh S, Papouchado BG, Burgart LJ, et al. Early hepatic stellate cell activation predicts severe hepatitis C recurrence after liver transplantation. Liver Transpl 2005;11:1207-1213.

20. Lau DT, Luxon BA, Xiao SY, et al. Intrahepatic gene expression profiles and alpha-smooth muscle actin patterns in hepatitis $C$ virus induced fibrosis. Hepatology 2005;42:273-281.

21. Calvaruso V, Di Marco V, Bavetta MG, et al. Quantification of fibrosis by collagen proportionate area predicts hepatic decompensation in hepatitis C cirrhosis. Aliment Pharmacol Ther 2015;41:477-486.

22. Tsochatzis $\mathrm{E}$, Bruno $\mathrm{S}$, Isgro $\mathrm{G}$, et al. Collagen proportionate area is superior to other histological methods for sub-classifying cirrhosis and determining prognosis. J Hepatol 2014;60:948-954.

23. Myers RP, Patel K, Pianko $S$, et al. The rate of fibrosis progression is an independent predictor of the response to antiviral therapy in chronic hepatitis C. J Viral Hepat 2003;10:16-22.

24. Russo MW, Firpi RJ, Nelson DR, et al. Early hepatic stellate cell activation is associated with advanced fibrosis after liver transplantation in recipients with hepatitis C. Liver Transpl 2005;11: $1235-1241$.
25. Mallat A, Lotersztajn S. Cellular mechanisms of tissue fibrosis. 5. Novel insights into liver fibrosis. Am J Physiol Cell Physiol 2013;305: C789-C799.

26. Pellicoro $A$, Ramachandran $P$, Iredale JP, et al. Liver fibrosis and repair: immune regulation of wound healing in a solid organ. Nat Rev Immunol 2014;14:181-194.

27. Xu L, Hui AY, Albanis E, et al. Human hepatic stellate cell lines, LX-1 and LX-2: new tools for analysis of hepatic fibrosis. Gut 2005:54:142-151.

28. Weiskirchen R, Weimer J, Meurer SK, et al. Genetic characteristics of the human hepatic stellate cell line LX-2. PLoS One 2013;8:e75692.

29. Ono M, Hatamochi A, Arakawa $M$, et al. Effects of cyclosporin $A$ on cell proliferation and collagen production by human skin fibroblasts. J Dermatol Sci 1991;2:274-280.

30. Ikeda H, Fujiwara K. Cyclosporin A and FK-506 in inhibition of rat Ito cell activation in vitro. Hepatology 1995;21:1161-1166.

31. Flores-Contreras L, Sandoval-Rodriguez AS, Mena-Enriquez MG, et al. Treatment with pirfenidone for two years decreases fibrosis, cytokine levels and enhances CB2 gene expression in patients with chronic hepatitis C. BMC Gastroenterol 2014;14:131.

32. Ibanez $\mathrm{P}$, Solis N, Pizarro M, et al. Effect of losartan on early liver fibrosis development in a rat model of nonalcoholic steatohepatitis. J Gastroenterol Hepatol 2007;22:846-851.

33. Bridle $K R$, Sobbe AL, de Guzman CE, et al. Lack of efficacy of mTOR inhibitors and ACE pathway inhibitors as antifibrotic agents in evolving and established fibrosis in Mdr2(-)/(-) mice. Liver Int 2015;35: 1451-1463.

34. Guillaud O, Gurram KC, Puglia M, et al. Angiotensin blockade does not affect fibrosis progression in recurrent hepatitis C after liver transplantation. Transplant Proc 2013;45:2331-2336.

35. Abu Dayyeh BK, Yang M, Dienstag JL, et al. The effects of angiotensin blocking agents on the progression of liver fibrosis in the HALT-C Trial cohort. Dig Dis Sci 2011;56:564-568.

36. Marshall RP, Simpson JK, Lukey PT. Strategies for biomarker discovery in fibrotic disease. Biochim Biophys Acta 2013;1832:1079-1087.

37. Bauer $\mathrm{Y}$, Tedrow J, de Bernard $\mathrm{S}$, et al. A novel genomic signature with translational significance for human idiopathic pulmonary fibrosis. Am J Respir Cell Mol Biol 2015;52:217-231. 